\title{
Robust portfolio choice with CVaR and VaR under distribution and mean return ambiguity
}

\author{
A. Burak Paç • Mustafa Ç. Pınar
}

Received: 8 July 2013/ Accepted: 16 October 2013

(C) Sociedad de Estadística e Investigación Operativa 2013

\begin{abstract}
We consider the problem of optimal portfolio choice using the Conditional Value-at-Risk (CVaR) and Value-at-Risk (VaR) measures for a market consisting of $n$ risky assets and a riskless asset and where short positions are allowed. When the distribution of returns of risky assets is unknown but the mean return vector and variance/covariance matrix of the risky assets are fixed, we derive the distributionally robust portfolio rules. Then, we address uncertainty (ambiguity) in the mean return vector in addition to distribution ambiguity, and derive the optimal portfolio rules when the uncertainty in the return vector is modeled via an ellipsoidal uncertainty set. In the presence of a riskless asset, the robust CVaR and VaR measures, coupled with a minimum mean return constraint, yield simple, mean-variance efficient optimal portfolio rules. In a market without the riskless asset, we obtain a closed-form portfolio rule that generalizes earlier results, without a minimum mean return restriction.
\end{abstract}

Keywords Robust portfolio choice $\cdot$ Ellipsoidal uncertainty · Conditional Value-at-Risk · Value-at-Risk · Distributional robustness

Mathematics Subject Classification (2010) $91 \mathrm{G} 10 \cdot 91 \mathrm{~B} 30 \cdot 90 \mathrm{C} 90$

\section{Introduction}

The Value-at-Risk (VaR) is widely used in the financial industry as a downside risk measure. Since VaR does not take into account the magnitude of potential losses, the Conditional Value-at-Risk (CVaR), defined as the mean losses in excess of VaR, was proposed as a remedy and results usually in convex (linear) portfolio

\footnotetext{
A. B. Paç · M. Ç. Pınar ( $)$

Deparment of Industrial Engineering, Bilkent University, 06800 Ankara, Turkey

e-mail: mustafap@bilkent.edu.tr
} 
optimization problems (Rockafellar and Uryasev 2000, 2002). The purpose of the present work is to give an explicit solution to the optimal portfolio choice problem by minimizing the $\mathrm{CVaR}$ and $\mathrm{VaR}$ measures under distribution and mean return ambiguity when short positions are allowed. Distribution ambiguity is understood in the sense that no knowledge of the return distribution for risky assets is assumed while the mean and variance/covariance are assumed to be known. The optimal portfolio choice problem using the aforementioned risk measures under distribution ambiguity and allowing short positions was studied by Chen et al. (2011) in a recent paper in the case of $n$ risky assets, extending the work of Zhu and Fukushima (2009) where the authors treat robust portfolio choice under distribution ambiguity. Chen et al. assumed that the mean return vector $\mu$ and variance-covariance matrix $\Gamma$ of risky assets are known, and compute portfolios that are robust in the sense that they minimize the worst-case CVaR risk measure over all distributions with fixed first and second moment information. They obtained closed-form robust optimal portfolio rules. The reader is referred to El Ghaoui et al. (2003), Natarajan et al. (2010) and Popescu (2005) for other related studies on portfolio optimization with distributional robustness, and to Tong et al. (2010) for a computational study of scenario-based $\mathrm{CVaR}$ in portfolio optimization. A recent reference work on portfolio optimization (using the mean-variance approach as well as semi-variance and utility functions) in both single and multi-period frameworks is by Steinbach (2001). In particular, Natarajan et al. (2010) study expected utility models in portfolio optimization under distribution ambiguity using a piecewise-linear concave utility function. They obtain bounds on the worst-case expected utility, and compute optimal portfolios by solving conic programs. They also relate their bounds to convex risk measures by defining a worst-case Optimized-CertaintyEquivalent (OCE) risk measure. It is well known that one of the two risk measures used in the present paper, namely $\mathrm{CVaR}$, can be obtained using the OCE approach for a class of utility functions; see Ben-Tal and Teboulle (2007). Thus the results of the present paper complement the previous work of Natarajan et al. (2010) by providing closed-form optimal portfolio rules for worst-case CVaR (and worst-case $\mathrm{VaR}$ ) under both distribution and mean return ambiguity. In the present paper, we first extend, in Sect. 2, the results of Chen et al. to the case where a riskless asset is also included in the asset universe, a case which is usually an integral part of optimal portfolio choice theory. The inclusion of the riskless asset in the asset universe leads to extreme positions in the portfolio, which implies that the robust $\mathrm{CVaR}$ and VaR measures as given in the present paper have to be utilized with a minimum mean return constraint in the presence of a riskless asset to yield closedform optimal portfolio rules. The distribution robust portfolios of Chen et al. (2011) are criticized in Delage and Ye (2010) for their sensitivity to uncertainties or estimation errors in the mean return data, a case that we refer to as Mean Return Ambiguity; see also Best and Grauer (1991a, b) and Black and Litterman (1992) for studies regarding sensitivity of optimal portfolios to estimation errors. To (partially) address this issue, we analyze in Sect. 3, the problem when the mean return is subject to ellipsoidal uncertainty (Ben-Tal and Nemirovski 1999, 1998; Garlappi et al. 2007; Goldfarb and Iyengar 2003; Ling and $\mathrm{Xu}$ 2012) in addition to distribution ambiguity, and derive a closed-form portfolio rule. The ellipsoidal 
uncertainty is regulated by a parameter that can be interpreted as a measure of confidence in the mean return estimate. In the presence of the riskless asset, a robust optimal portfolio rule under distribution and mean return ambiguity is obtained if the quantile parameter of $\mathrm{CVaR}$ or $\mathrm{VaR}$ measures is above a threshold depending on the optimal Sharpe ratio of the market and the confidence regulating parameter, or no such optimal rule exists (the problem is infeasible). The key to obtain optimal portfolio rules in the presence of a riskless under distribution and mean return ambiguity asset is again to include a minimum mean return constraint to trace the efficient robust CVaR (or robust VaR) frontier; Steinbach (2001). The incremental impact of adding robustness against mean return ambiguity in addition to distribution ambiguity is to alter the optimal Sharpe ratio of the market viewed by the investor. The investor views a smaller optimal Sharpe ratio decremented by the parameter reflecting the confidence of the investor in the mean return estimate.

In the case the riskless asset is not included in the portfolio problem, in Sect. 4, we derive in closed form the optimal portfolio choice robust against distribution and ellipsoidal mean return ambiguity without using a minimum mean return constraint, which generalizes a result of Chen et al. (2011) stated in the case of distribution ambiguity only, i.e., full confidence in the mean return estimate.

\section{Minimizing robust $\mathrm{CVaR}$ and $\mathrm{VaR}$ in the presence of a riskless asset under distribution ambiguity}

We work in a financial market with a riskless asset with return rate $R$ in addition to $n$ risky assets. We investigate robust solutions minimizing $\mathrm{CVaR}$ and $\mathrm{VaR}$ measures. The unit initial wealth is allocated into the riskless and risky assets so as to allow short positions, thus we can define the loss function as a function of the vector $x \in \mathbb{R}^{n}$ of allocations to $n$ risky assets and the random vector $\xi$ of return rates for these assets:

$$
f(x, \xi)=-\left(x^{\mathrm{T}} \xi+R\left(1-x^{\mathrm{T}} e\right)\right),
$$

where $e$ denotes an $n$-vector of all ones. For the calculation of CVaR and VaR, we use the method in Rockafellar and Uryasev (2000), minimizing over $\gamma$ the auxiliary function:

$$
F_{\theta}(x, \gamma)=\gamma+\frac{1}{1-\theta} \mathbb{E}\left[(f(x, \xi)-\gamma)_{+}\right]
$$

i.e., the $\theta-\mathrm{CVaR}$ is calculated as follows:

$$
\mathrm{CVaR}_{\theta}(x)=\min _{\gamma \in \mathbb{R}} F_{\theta}(x, \gamma),
$$

where $\theta$ is the threshold probability level or the quantile parameter, which is generally taken to be in the interval $[0.95,1)$. The convex set consisting of $\gamma$ values that minimize $F_{\theta}$ contains the $\theta-\mathrm{VaR}, \operatorname{VaR}_{\theta}(x)$, which is the minimum value in the set. 
The worst-case $\mathrm{CVaR}$, when $\xi$ may assume a distribution from the set $\mathbb{D}=\left\{\pi \mid \mathbb{E}_{\pi}[\xi]=\mu, \operatorname{Cov}_{\pi}[\xi]=\Gamma \succ 0\right\}$ [i.e., the set of all distributions with (known and/or trusted) mean $\mu$ and covariance $\Gamma]$, is defined as follows:

$$
\begin{gathered}
\operatorname{RCVaR}_{\theta}(x)=\max _{\pi \in \mathbb{D}} \operatorname{CVaR}_{\theta}(x) \\
=\max _{\pi \in \mathbb{D}} \min _{\gamma \in \mathbb{R}} F_{\theta}(x, \gamma) .
\end{gathered}
$$

For convenience, in the rest of the paper, we replace occurrences of the sup operator with max since in all cases we consider the sup is attained.

We assume $\mu$ is not a multiple of $e$, as usual. Let us define the excess mean return $\tilde{\mu}=\mu-R e$, and the highest attainable Sharpe ratio in the market $H=\tilde{\mu}^{\mathrm{T}} \Gamma^{-1} \tilde{\mu}$; see Best (2010). The following Theorem ${ }^{1}$ gives an explicit solution of the portfolio choice problem of minimizing worst-case $\mathrm{CVaR}$ under a minimum mean return constraint

$$
\min _{x \in \mathbb{R}^{n}:(\mu-R e)^{\mathrm{T}} x \geq d-R} \operatorname{RCVaR}_{\theta}(x),
$$

where $d \in \mathbb{R}_{+}$is a minimum target mean return parameter. We assume the minimum mean target return is larger than the riskless return, i.e., $d>R$.

Theorem 1 For $\theta \geq \frac{H}{H+1}$, the problem Eq. (6) admits the optimal portfolio rule

$$
x^{*}=\frac{d-R}{H} \Gamma^{-1} \tilde{\mu} .
$$

For $\theta<\frac{H}{H+1}$, the problem Eq. (6) is unbounded.

Proof Since the set of distributions $\mathbb{D}$ is convex and the function $\gamma+\frac{1}{1-\theta} \mathbb{E}\left[(f(x, \xi)-\gamma)_{+}\right]$is convex in $\gamma$ for every $\xi$, we can interchange supremum and minimum; see Theorem 2.4 of Shapiro (2011). More precisely, one considers first the problem $\min _{\gamma \in \mathbb{R}} \max _{\pi \in \mathbb{D}} F_{\theta}(x, \gamma)$ and one finds a unique optimal solution as we shall do below. Then, using convexity of $\mathbb{D}$ and the convexity of the function $\gamma+\frac{1}{1-\theta} \mathbb{E}\left[(f(x, \xi)-\gamma)_{+}\right]$in $\gamma$ one invokes Theorem 2.4 of Shapiro (2011) that allows interchange of min and max. We carry out this sequence of operations below:

$$
\begin{aligned}
\min _{\gamma \in \mathbb{R}} \max _{\pi \in \mathbb{D}} F_{\theta}(x, \gamma) & =\min _{\gamma \in \mathbb{R}} \gamma+\frac{1}{1-\theta} \max _{\pi \in \mathbb{D}} \mathbb{E}\left[\left(-x^{\mathrm{T}} \xi-R+R x^{\mathrm{T}} e-\gamma\right)_{+}\right] \\
& =\min _{\gamma \in \mathbb{R}} \gamma+\frac{1}{1-\theta} \max _{\xi \sim(\mu, \Gamma)} \mathbb{E}\left[\left(-R-\gamma-x^{\mathrm{T}}(\xi-R e)\right)_{+}\right] \\
& =\min _{\gamma \in \mathbb{R}} \gamma+\frac{1}{1-\theta} \max _{\eta \sim\left(v, \sigma^{2}\right)} \mathbb{E}\left[(-R-\gamma-\eta)_{+}\right]
\end{aligned}
$$

\footnotetext{
1 While our proof is similar to the proof in Chen et al. (2011) in essence, their proof is faulty because their argument for exchanging max and min relies on a result of Zhu and Fukushima (2009) which is valid for discrete distributions. Our setting here, like that of Chen et al. (2011) is not confined to discrete distributions. Hence, a different justification is needed for exchanging max and min.
} 


$$
=\min _{\gamma \in \mathbb{R}} \gamma+\frac{1}{1-\theta} \frac{-R-\gamma-v+\sqrt{\sigma^{2}+(-R-\gamma-v)^{2}}}{2} .
$$

Maximum operators in Eqs. (7) and (8) are equivalent, since by a slight modification of Lemma 2.4 in Chen et al. (2011) we can state the equivalence of following sets of univariate random distributions:

$$
\begin{aligned}
& \mathbb{D}_{1}=\left\{x^{\mathrm{T}}(\xi-R e): \xi \sim(\mu, \Gamma)\right\} \\
& \mathbb{D}_{2}=\left\{\eta: \eta \sim\left(v, \sigma^{2}\right)\right\},
\end{aligned}
$$

where $v=x^{\mathrm{T}}(\mu-R e)$, and $\sigma^{2}=x^{\mathrm{T}} \Gamma x$. Equality Eq. (9) follows by Lemma 2.2 of Chen et al. (2011). Then $\operatorname{RCVaR}_{\theta}(x)$ is the minimization of the following function over $\gamma$ :

$$
h_{x}(\gamma)=\gamma+\frac{1}{1-\theta} \frac{-R-\gamma-v+\sqrt{\sigma^{2}+(R+\gamma+v)^{2}}}{2} .
$$

$\gamma_{x}^{*}$ minimizing $h_{x}(\gamma)$ can be found by equating the first derivative to zero:

$$
h_{x}^{\prime}(\gamma)=1+\frac{1}{1-\theta} \frac{-1+(R+\gamma+v)\left(\sigma^{2}+(R+\gamma+v)^{2}\right)^{-\frac{1}{2}}}{2} .
$$

Hence, we have

$$
2 \theta-1=(R+\gamma+v) / \sqrt{\sigma^{2}+(R+\gamma+v)^{2}},
$$

(recall that $\theta>0.5$ so that $2 \theta-1>0$ ) or, equivalently,

$$
(2 \theta-1)^{2}\left(\sigma^{2}+(R+\gamma+v)^{2}\right)=(R+\gamma+v)^{2},
$$

or,

$$
(2 \theta-1)^{2} \sigma^{2}=(R+\gamma+v)^{2}\left(1-(2 \theta-1)^{2}\right)
$$

whence we obtain

$$
\begin{aligned}
R+\gamma+v & =\frac{(2 \theta-1) \sigma}{\sqrt{4 \theta-4 \theta^{2}}} \\
\gamma_{x}^{*} & =-R-v+\frac{(2 \theta-1) \sigma}{2 \sqrt{\theta} \sqrt{1-\theta}},
\end{aligned}
$$

since $h_{x}(\gamma)$ is a convex function as is verified immediately using the second derivative test:

$$
h_{x}^{\prime \prime}(\gamma)=\frac{1}{2(1-\theta)}\left(\sigma^{2}+(R+\gamma+v)^{2}\right)^{-\frac{3}{2}} \sigma^{2} \geq 0 .
$$

The minimum value of $h_{x}(\gamma)$ being known, we can calculate the min max value: 


$$
\begin{aligned}
\min _{\gamma \in \mathbb{R}} \max _{\pi \in \mathbb{D}} F_{\theta}(x, \gamma) & =\min _{\gamma \in \mathbb{R}} h_{x}(\gamma) \\
& =h_{x}\left(\gamma_{x}^{*}\right) \\
& =-R-v+\frac{(2 \theta-1) \sigma}{2 \sqrt{\theta} \sqrt{1-\theta}}+\frac{1}{1-\theta} \frac{\frac{-(2 \theta-1) \sigma}{2 \sqrt{\theta} \sqrt{1-\theta}}+\sqrt{\sigma^{2}+\frac{(2 \theta-1)^{2}}{4 \theta(1-\theta)}} \sigma^{2}}{2} \\
& =-R-v+\frac{(2 \theta-1) \sigma}{2 \sqrt{\theta} \sqrt{1-\theta}}+\frac{1}{1-\theta} \frac{\sigma}{2}\left(\frac{-2 \theta+1+1}{2 \sqrt{\theta} \sqrt{1-\theta}}\right) \\
& =-R-v+\frac{(2 \theta-1) \sigma}{2 \sqrt{\theta} \sqrt{1-\theta}}+\frac{1}{1-\theta} \frac{\sigma(1-\theta)}{2 \sqrt{\theta} \sqrt{1-\theta}} \\
& =-R-v+\frac{2 \theta \sigma}{2 \sqrt{\theta} \sqrt{1-\theta}} \\
& =-R-v+\frac{\sqrt{\theta}}{\sqrt{1-\theta}} \sigma \\
& =-R-(\mu-\operatorname{Re})^{\mathrm{T}} x+\frac{\sqrt{\theta}}{\sqrt{1-\theta}} \sqrt{x^{\mathrm{T}} \Gamma x} .
\end{aligned}
$$

Hence, by Theorem 2.4 of (Shapiro 2011) we have

$$
\operatorname{RCVaR}_{\theta}(x)=-R-(\mu-R e)^{\mathrm{T}} x+\frac{\sqrt{\theta}}{\sqrt{1-\theta}} \sqrt{x^{\mathrm{T}} \Gamma x} .
$$

Minimizing the above expression for worst-case CVaR under the minimum mean return constraint, the robust optimal portfolio selection can be found ${ }^{2}$.Using a nonnegative multiplier $\lambda$, the Lagrange function is

$$
L(x, \lambda)=-R-(\mu-R e)^{\mathrm{T}} x+\frac{\sqrt{\theta}}{\sqrt{1-\theta}} \sqrt{x^{\mathrm{T}} \Gamma x}+\lambda\left(d-R-(\mu-R e)^{\mathrm{T}} x\right) .
$$

The first-order conditions are:

$$
-\mu+R e+\frac{\kappa \Gamma x}{\sqrt{x^{\mathrm{T}} \Gamma x}}-\lambda(\mu-R e)=0,
$$

where we defined $\kappa \equiv \frac{\sqrt{\theta}}{\sqrt{1-\theta}}$ for convenience. We make the supposition that $x \neq 0$ and define $\sigma \equiv \sqrt{x^{\mathrm{T}} \Gamma x}$. We get the candidate solution

$$
x_{\mathrm{c}}=\frac{\sigma(\lambda+1)}{\kappa} \Gamma^{-1} \tilde{\mu} .
$$

Now, using the identity $x_{\mathrm{c}}^{\mathrm{T}} \Gamma x_{\mathrm{c}}=\sigma^{2}$, we obtain the equation

\footnotetext{
${ }^{2}$ It is a simple exercise to show that in the absence of the minimum mean return constraint the portfolio position in the $i$ th risky asset tends to $\pm \infty$ depending on the sign of the $i$ th component of $\tilde{\Gamma}^{-1} \tilde{\mu}$.
} 


$$
(\lambda+1)^{2}=\frac{\kappa^{2}}{H},
$$

which implies that $\lambda=\frac{\kappa}{\sqrt{H}}-1$. Under the condition $\frac{\kappa}{\sqrt{H}} \geq 1$ we ensure $\lambda \geq 0$. Now, utilizing the constraint which we assumed would be tight, the resulting equation yields (after substituting for $x_{\mathrm{c}}$ and $\lambda$ )

$$
\sigma=\frac{d-R}{\sqrt{H}}
$$

which is positive under the assumption $d>R$ ( $H$ is positive by positive definiteness of $\Gamma$ and the assumption that $\mu$ is not a multiple of $e$ ). Now, substitute the above expressions for $\sigma$ and $\lambda$ into $x_{\mathrm{c}}$, and the desired expression is obtained after evident simplification. If $\frac{\kappa}{\sqrt{H}}<1$ then the problem Eq. (6) is unbounded by the convex duality theorem since it is always feasible.

The VaR measure can also be calculated using the auxiliary function Eq. (2):

$$
\operatorname{VaR}_{\theta}(x)=\arg \min _{\gamma \in \mathbb{R}} F_{\theta}(x, \gamma) .
$$

The worst-case VaR is now calculated as follows:

$$
\begin{aligned}
\operatorname{RVaR}_{\theta}(x) & =\max _{\pi \in \mathbb{D}} \operatorname{VaR}_{\theta}(x) \\
& =\max _{\pi \in \mathbb{D}} \arg \min _{\gamma \in \mathbb{R}} F_{\theta}(x, \gamma) \\
& =\arg \min _{\gamma \in \mathbb{R}} \max _{\pi \in \mathbb{D}} F_{\theta}(x, \gamma) \\
& =\arg \min _{\gamma \in \mathbb{R}} h_{x}(\gamma) \\
& =\gamma_{x}^{*} .
\end{aligned}
$$

Again, a change of the order of operators makes this calculation possible by Theorem 2.4 of Shapiro (2011). With a similar approach to that followed for CVaR, we pose the problem of minimizing the robust VaR:

$$
\min _{x \in \mathbb{R}^{n}}-R-x^{\mathrm{T}} \mu+R x^{\mathrm{T}} e+\frac{(2 \theta-1)}{2 \sqrt{\theta} \sqrt{1-\theta}} \sqrt{x^{\mathrm{T}} \Gamma x}
$$

subject to

$$
(\mu-R e)^{\mathrm{T}} x \geq d-R .
$$

We obtain a replica of the previous result in this case. Hence the proof, which is identical, is omitted.

Theorem 2 For $\theta \geq \frac{1}{2}+\frac{1}{2} \frac{\sqrt{H}}{\sqrt{H+1}}$, the problem of minimizing robust VaR under distribution ambiguity and a minimum mean return restriction admits the optimal portfolio rule

$$
x^{*}=\frac{d-R}{H} \Gamma^{-1} \tilde{\mu} .
$$


For $\theta<\frac{1}{2}+\frac{1}{2} \frac{\sqrt{H}}{\sqrt{H+1}}$, the problem is unbounded.

In both results stated above, the optimal portfolios are mean-variance efficient. We plot the critical thresholds for $\theta$ given in Theorems 1 and 2 in Fig. 1 below. Both thresholds tend to one as $H$ goes to infinity and the threshold curve for robust $\mathrm{VaR}$ dominates that of robust $\mathrm{CVaR}$. Hence, robust $\mathrm{VaR}$ leads to aggressive portfolio behavior in a larger interval for $\theta$ than robust CVaR. In other words, one has to choose a larger confidence level $\theta$ for robust $\mathrm{VaR}$ as compared to robust $\mathrm{CVaR}$ to make an optimal portfolio choice. Thus we can affirm that robust VaR is more conservative than robust $\mathrm{CVaR}$.

Based on the above results, it is straightforward to derive the equations of the robust $\mathrm{CVaR}$ efficient frontier and the robust $\mathrm{VaR}$ efficient frontier. Both robust frontiers are the straight lines governed by the equation

$$
d=\frac{\kappa}{\kappa-\sqrt{H}} R+\frac{\sqrt{H}}{\kappa-\sqrt{H}} f,
$$

where for $f=\mathrm{RCVaR}$ and $\kappa=\frac{\sqrt{\theta}}{\sqrt{1-\theta}}$ we obtain the efficient frontier for robust $\mathrm{CVaR}$; and for $f=\mathrm{RVaR}$ and $\kappa=\frac{(2 \theta-1)}{2 \sqrt{\theta} \sqrt{1-\theta}}$ we get the robust $\mathrm{VaR}$ efficient frontier.

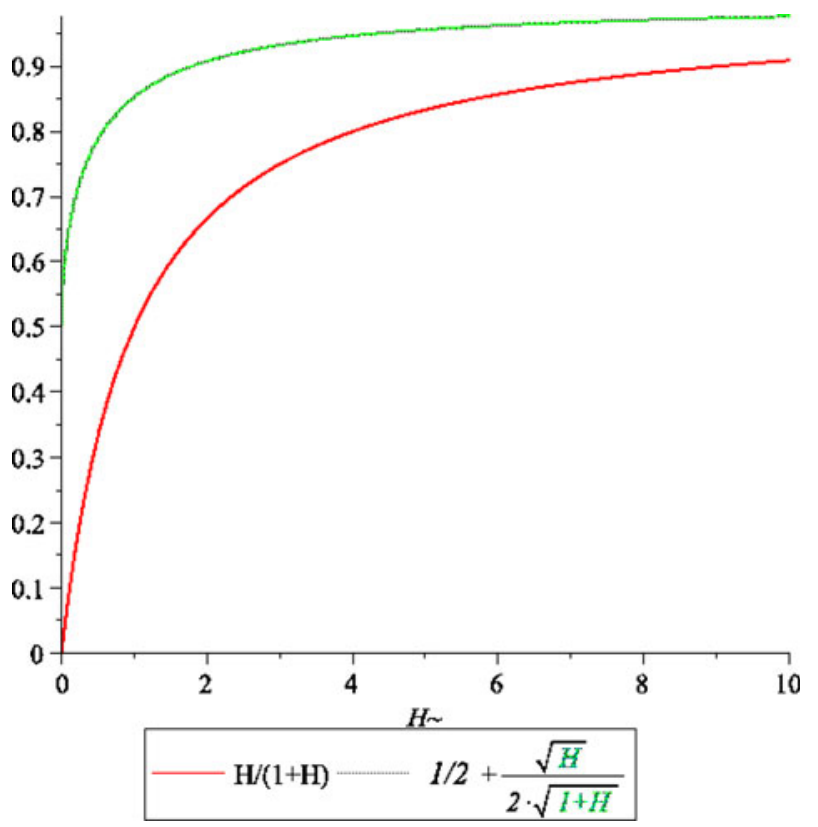

Fig. 1 The critical thresholds for robust $\mathrm{CVaR}$ and robust VaR. The upper curve is the threshold value curve for robust $\mathrm{VaR}$ 


\section{Robust CVaR in the presence of riskless asset under distribution and mean return ambiguity}

We consider now the problem of choosing a portfolio $x \in \mathbb{R}^{n}$ that minimizes the function

$$
\operatorname{RRCVaR}_{\theta}(x)=\max _{\pi \in \mathbb{D}, \bar{\mu} \in U_{\bar{\mu}}} \operatorname{CVaR}_{\theta}(x)
$$

or equivalently

$$
\operatorname{RRCVaR}_{\theta}(x)=\max _{\bar{\mu} \in U_{\bar{\mu}}} \max _{\pi \in \mathbb{D}} \min _{\gamma \in \mathbb{R}} F_{\theta}(x, \gamma)
$$

where we define the ellipsoidal uncertainty set $U_{\bar{\mu}}=\left\{\bar{\mu} \mid\left\|\Gamma^{-1 / 2}\left(\bar{\mu}-\mu^{\mathrm{nom}}\right)\right\|_{2} \leq \sqrt{\epsilon}\right\}$ for the mean return denoted $\bar{\mu}$, where $\mu^{\text {nom }}$ denotes a nominal mean return vector which can be considered as the available estimate of mean return. The parameter $\epsilon$ acts as a measure of confidence in the mean return estimate. We consider now the problem of choosing a portfolio $x \in \mathbb{R}^{n}$ that minimizes the function

$$
\operatorname{RRCVaR}_{\theta}(x)=\max _{\bar{\mu} \in U_{\bar{\mu}}} \max _{\pi \in \mathbb{D}} \min _{\gamma \in \mathbb{R}} F_{\theta}(x, \gamma)
$$

subject to

$$
(\bar{\mu}-R e)^{\mathrm{T}} x \geq d-R .
$$

As in the previous section we define $\mathbb{D}=\left\{\pi \mid \mathbb{E}_{\pi}[\xi]=\bar{\mu}, \operatorname{Cov}_{\pi}[\xi]=\Gamma \succ 0\right\}$. Considering the inner max min problem over $\pi \in \mathbb{D}$ and $\gamma \in \mathbb{R}$, respectively, as in the proof of Theorem 1 of previous section while we keep $\bar{\mu}$ fixed, we arrive at the inner problem for the objective function (recall Eq. 10) that we can transform immediately:

$$
\begin{aligned}
\max _{\bar{\mu} \in U_{\bar{\mu}}} \operatorname{RCVaR}_{\theta}(x) & =\max _{\bar{\mu} \in U_{\bar{\mu}}}-R-(\bar{\mu}-R e)^{\mathrm{T}} x+\frac{\sqrt{\theta}}{\sqrt{1-\theta}} \sqrt{x^{\mathrm{T}} \Gamma x} \\
& =\max _{\bar{\mu} \in U_{\bar{\mu}}}-R-(\bar{\mu}-R e)^{\mathrm{T}} x+\frac{\sqrt{\theta}}{\sqrt{1-\theta}} \sqrt{x^{\mathrm{T}} \Gamma x} \\
& =-R-\left(\mu^{*}\right)^{\mathrm{T}} x+\sqrt{\epsilon} \sqrt{x^{\mathrm{T}} \Gamma x}+\frac{\sqrt{\theta}}{\sqrt{1-\theta}} \sqrt{x^{\mathrm{T}} \Gamma x}
\end{aligned}
$$

where the last equality follows using a well-known transformation result in robust optimization (see e.g., Ben-Tal and Nemirovski 1999), and $\mu^{*}=\mu^{\text {nom }}-R e$.

Using the same transformation on the minimum mean return constraint as well, we obtain the second-order conic problem

$$
\min _{x \in \mathbb{R}^{n}}-R-x^{\mathrm{T}} \mu^{*}+\left(\sqrt{\epsilon}+\frac{\sqrt{\theta}}{\sqrt{1-\theta}}\right) \sqrt{x^{\mathrm{T}} \Gamma x}
$$

subject to

$$
\left(\mu^{*}\right)^{\mathrm{T}} x-\sqrt{\epsilon} \sqrt{x^{\mathrm{T}} \Gamma x} \geq d-R,
$$

where $d>R$. 


\section{Theorem 3}

1. Under the Slater constraint qualification, if $\theta>\frac{(\sqrt{H}-\sqrt{\epsilon})^{2}}{(\sqrt{H}-\sqrt{\epsilon})^{2}+1}$, and $\epsilon<H$ then the problem Eq. (14) admits the optimal portfolio rule

$$
x^{*}=\frac{d-R}{(\sqrt{H}-\sqrt{\epsilon}) \sqrt{H}} \Gamma^{-1} \tilde{\mu} .
$$

2. If $\epsilon=H$, the problem is unbounded.

3. If $\epsilon>H$, the problem is infeasible.

Proof Under the Slater constraint qualification the Karush-Kuhn-Tucker optimality conditions are both necessary and sufficient. Using a non-negative multiplier $\lambda$ we have the Lagrange function

$$
\begin{aligned}
L(x, \lambda)= & -R-x^{\mathrm{T}} \mu^{*}+\left(\sqrt{\epsilon}+\frac{\sqrt{\theta}}{\sqrt{1-\theta}}\right) \sqrt{x^{\mathrm{T}} \Gamma x}+\lambda\left(d-R-\left(\mu^{*}\right)^{\mathrm{T}} x\right. \\
& \left.+\sqrt{\epsilon} \sqrt{x^{\mathrm{T}} \Gamma x}\right) .
\end{aligned}
$$

Going through the usual steps as in the proof of Theorem 1 under the supposition that $\sigma$ (defined as $\sqrt{x^{\mathrm{T}} \Sigma x}$ ) is a finite non-zero positive number, we have the candidate solution:

$$
x_{\mathrm{c}}=\frac{\sigma(\lambda+1)}{\kappa+\sqrt{\epsilon}(\lambda+1)} \Gamma^{-1} \mu^{*} .
$$

From the identity $x_{\mathrm{c}}^{\mathrm{T}} \Gamma x_{\mathrm{c}}=\sigma^{2}$ we obtain the quadratic equation in $\lambda$ :

$$
(H-\epsilon) \lambda^{2}+(2 H-2 \epsilon-2 \sqrt{\epsilon} \kappa) \lambda+H-\epsilon-2 \sqrt{\epsilon} \kappa-\kappa^{2}=0
$$

with the two roots

$$
-\frac{\kappa+\sqrt{H}+\sqrt{\epsilon}}{\sqrt{H}+\sqrt{\epsilon}},-\frac{-\kappa+\sqrt{H}-\sqrt{\epsilon}}{\sqrt{H}-\sqrt{\epsilon}} .
$$

The left root cannot be positive, so it is discarded. The right root is positive if $H>\epsilon$ and $\kappa \geq \sqrt{H}-\sqrt{\epsilon}$. Now, returning to the conic constraint which is assumed to be tight we obtain an equation in $\sigma$ after substituting for $\lambda$ in the right root, and solving for $\sigma$ using straightforward algebraic simplification we get

$$
\sigma=\frac{d-R}{\sqrt{H}-\sqrt{\epsilon}},
$$

which is positive provided $H>\epsilon$. Now, the expression for $x^{*}$ is obtained after substitution for $\sigma$ and $\lambda$ into $x_{\mathrm{c}}$ and evident simplification.

Part 2 is immediate from the result of Part 1.

For part 3, if $\epsilon \geq H$, our hypothesis of a finite positive non-zero $\sigma$ is false, in which case the only possible value for $\sigma$ is zero, achieved at the zero risky portfolio which is infeasible. $\square$ 
The problem of minimizing the robust VaR under distribution and mean return ambiguity in the presence of a minimum target mean return constraint is posed as

$$
\min _{x \in \mathbb{R}^{n}}-R-x^{\mathrm{T}} \mu^{*}+\left(\sqrt{\epsilon}+\frac{(2 \theta-1)}{2 \sqrt{\theta} \sqrt{1-\theta}}\right) \sqrt{x^{\mathrm{T}} \Gamma x}
$$

subject to

$$
\left(\mu^{*}\right)^{\mathrm{T}} x-\sqrt{\epsilon} \sqrt{x^{\mathrm{T}} \Gamma x} \geq d-R .
$$

Again, we obtain a result similar to the previous theorem in this case. The proof is a verbatim repetition of the proof of the previous theorem, hence omitted.

\section{Theorem 4}

1. Under the Slater constraint qualification, if $\theta>\frac{1}{2}+\frac{\sqrt{H}-\sqrt{\epsilon}}{2 \sqrt{1+(\sqrt{H}-\sqrt{\epsilon})^{2}}}$, and $\epsilon<H$ then the problem of minimizing the robust VaR under distribution and mean return ambiguity in the presence of a minimum target mean return constraint admits the optimal portfolio rule

$$
x^{*}=\frac{d-R}{(\sqrt{H}-\sqrt{\epsilon}) \sqrt{H}} \Gamma^{-1} \tilde{\mu} .
$$

2. If $\epsilon=H$, the problem is unbounded.

3. If $\epsilon>H$, the problem is infeasible.

Notice that we obtain identical and mean-variance efficient portfolio rules for both $\mathrm{CVaR}$ and VaR under distribution and mean return ambiguity. Furthermore, the optimal portfolio rules reduce to those of Theorems 1 and 2, respectively, for $\epsilon=0$, the case of distribution ambiguity only.

The robust $\mathrm{CVaR}$ and $\mathrm{VaR}$ efficient frontiers are straight lines given by the equation:

$$
d=\frac{\kappa}{\kappa-\sqrt{H}+\sqrt{\epsilon}} R+\frac{\sqrt{H}-\sqrt{\epsilon}}{\kappa-\sqrt{H}+\sqrt{\epsilon}} f,
$$

where for $f=\mathrm{RRCVaR}$ and $\kappa=\frac{\sqrt{\theta}}{\sqrt{1-\theta}}$ we obtain the efficient frontier for robust CVaR; and for $f=\mathrm{RRVaR}$ and $\kappa=\frac{(2 \theta-1)}{2 \sqrt{\theta} \sqrt{1-\theta}}$ we get the robust VaR efficient frontier.

Comparing the above results and efficient frontier to the results of the previous section and to the efficient frontier Eq. (11) for the case of ambiguity distribution only, we notice that the effect of introducing mean return ambiguity in addition to distribution ambiguity has the effect of replacing $\sqrt{H}$ by $\sqrt{H}-\sqrt{\epsilon}$. More precisely, the mean return ambiguity decreases the optimal Sharpe ratio of the market viewed by the investor. The investor can form an optimal portfolio in the risky assets as long as his/her confidence in the mean return vector is not too low, i.e., his $\epsilon$ does not exceed the optimal Sharpe ratio of the market. 
The efficient frontier line for the case of robust portfolios in the face of both distribution and mean return ambiguity is less steep than the efficient robust portfolios for distribution ambiguity only. This simple fact can be verified by direct computation:

$$
\frac{\sqrt{H}-\sqrt{\epsilon}}{\kappa-\sqrt{H}+\sqrt{\epsilon}}-\frac{\sqrt{H}}{\kappa-\sqrt{H}}=-\frac{\kappa \sqrt{\epsilon}}{(\kappa-\sqrt{H})(\kappa-\sqrt{H}+\sqrt{\epsilon})}<0 .
$$

The $d$-intercept for the former is also smaller than for the latter as can be easily seen. We provide a numerical illustration in Fig. 2 with $H=0.47222, \epsilon=0.4, R=1.01$ and $\theta=0.95$. The efficient portfolios robust to distribution ambiguity are represented by the steeper line. It is clear that the incremental effect of mean return ambiguity and robustness is to render the investor more risk averse and more cautious.

\section{Without the riskless asset}

We consider now the problems of the previous section as treated in Chen et al. (2011), i.e., without the riskless asset and without the minimum mean return constraint since we do not need this restriction to obtain an optimal portfolio rule. In that case we are dealing with the loss function:

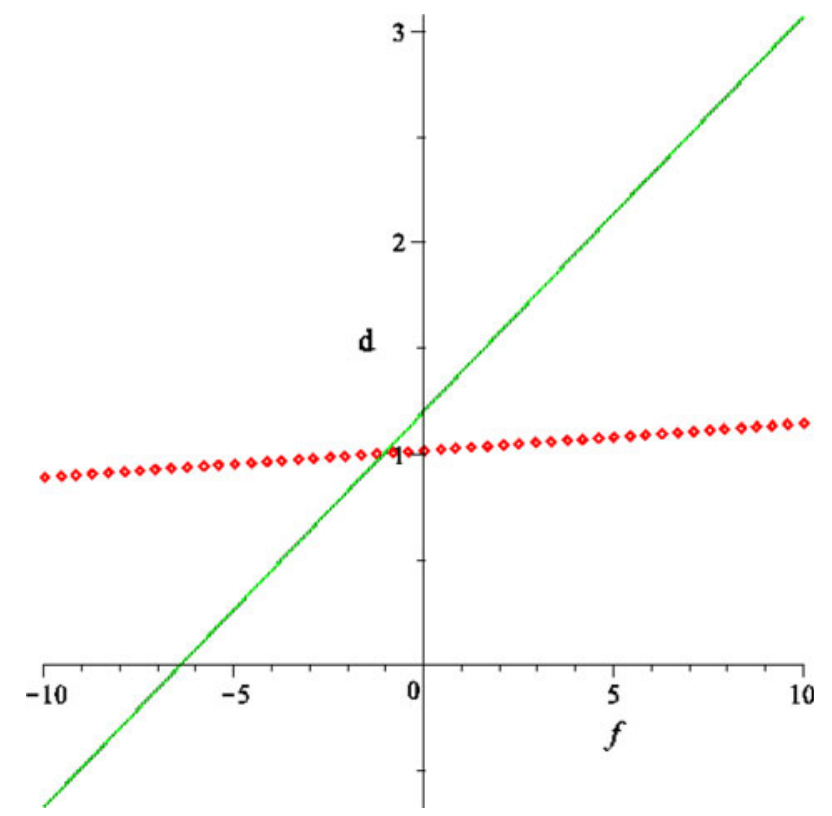

Fig. 2 The efficient frontier lines for robust $\mathrm{CVaR}$ and robust $\mathrm{VaR}$ for $H=0.47222, \epsilon=0.4, R=1.01$ and $\theta=0.95$. The steeper line corresponds to distribution ambiguity case while the point line corresponds to distribution and mean return ambiguity case 


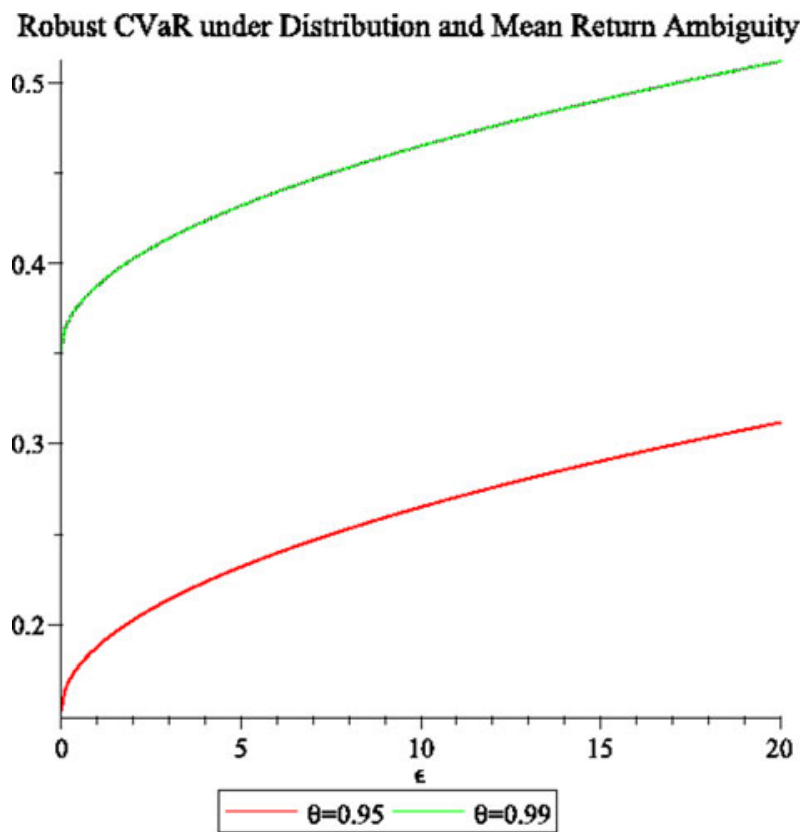

Fig. 3 The behavior of robust CVaR as a function of $\epsilon$ in the case without the riskless asset. The upper curve is for $\theta=0.99$ and the lower curve is for $\theta=0.95$

$$
f(x, \xi)=-x^{\mathrm{T}} \xi
$$

and the auxiliary function

$$
F_{\theta}(x, \gamma)=\gamma+\frac{1}{1-\theta} \mathbb{E}\left[\left(-x^{\mathrm{T}} \xi-\gamma\right)_{+}\right]
$$

In Chen et al. (2011) Theorem 2.9 the authors solve the problem

$$
\min _{x \in \mathbb{R}^{n}: e^{T} x=1} \max _{\pi \in \mathbb{D}} \operatorname{CVaR}_{\theta}(x)
$$

in closed form. We shall now attack the problem under the assumption that the mean returns are subject to errors that we confine to the ellipsoid: $U_{\bar{\mu}}=\left\{\bar{\mu} \mid \| \Gamma^{-1 / 2}(\bar{\mu}-\right.$ $\left.\left.\mu^{\text {nom }}\right) \|_{2} \leq \sqrt{\epsilon}\right\}$ for the mean return denoted $\bar{\mu}$, where $\mu^{\text {nom }}$ denotes a nominal mean return vector as in the previous section. That is, we are interested in solving

$$
\min _{x \in \mathbb{R}^{n}: e^{T} x=1} \max _{\bar{\mu} \in U_{\bar{\mu}}} \max _{\pi \in \mathbb{D}} \operatorname{CVaR}_{\theta}(x) .
$$

Using partially the proof of Theorem 2.9 in Chen et al. (2011), and Theorem 2.4 of Shapiro (2011) as in the previous section, we have for arbitrary $\bar{\mu}$

$$
\max _{\pi \in \mathbb{D}} \operatorname{CVaR}_{\theta}(x)=\sqrt{\frac{\theta}{1-\theta}} \sqrt{x^{\mathrm{T}} \Gamma x}-x^{\mathrm{T}} \bar{\mu},
$$

and the maximum is attained at 


$$
\frac{2 \theta-1}{2 \sqrt{\theta(1-\theta)}} \sqrt{x^{\mathrm{T}} \Gamma x}-x^{\mathrm{T}} \bar{\mu}
$$

which happens to be equal to the robust VaR under distribution ambiguity. Therefore, we obtain

$$
\max _{\bar{\mu} \in U_{\bar{\mu}}} \max _{\pi \in \mathbb{D}} \operatorname{CVaR}_{\theta}(x)=\sqrt{\frac{\theta}{1-\theta}} \sqrt{x^{\mathrm{T}} \Gamma x}-x^{\mathrm{T}} \mu^{\mathrm{nom}}+\sqrt{\epsilon} \sqrt{x^{\mathrm{T}} \Gamma x} .
$$

Now, we are ready to process the problem

$$
\min _{x \in \mathbb{R}^{n}: e^{\mathrm{T}} x=1} \sqrt{\frac{\theta}{1-\theta}} \sqrt{x^{\mathrm{T}} \Gamma x}-x^{\mathrm{T}} \mu^{\mathrm{nom}}+\sqrt{\epsilon} \sqrt{x^{\mathrm{T}} \Gamma x} .
$$

From the first-order conditions we obtain

$$
x=\frac{\sigma}{\kappa+\sqrt{\epsilon}} \Gamma^{-1}\left(\mu^{\mathrm{nom}}+\lambda e\right)
$$

where $\sigma \equiv\left(x^{\mathrm{T}} \Gamma x\right)^{1 / 2}, \kappa=\sqrt{\theta /(1-\theta)}$ and $\lambda$ is the Lagrange multiplier. Using the equation $\sigma^{2}=x^{\mathrm{T}} \Gamma x$ we obtain the quadratic equation:

$$
C \lambda^{2}+2 B \lambda+A-(\kappa+\sqrt{\epsilon})^{2}=0
$$

where $A=\left(\mu^{\text {nom }}\right)^{\mathrm{T}} \Gamma^{-1} \mu^{\text {nom }}, C=e^{\mathrm{T}} \Gamma^{-1} e, B=e^{\mathrm{T}} \Gamma^{-1} \mu^{\text {nom }}$. We solve for $\lambda$ under the condition

$$
C(\kappa+\sqrt{\epsilon})^{2}>A C-B^{2}
$$

(note that $A C-B^{2}>0$ by Cauchy-Schwarz inequality):

$$
\lambda=\frac{-B+\sqrt{B^{2}-A C+C(\kappa+\sqrt{\epsilon})^{2}}}{C} .
$$

We discard the root $\frac{-B-\sqrt{B^{2}-A C+C(\kappa+\sqrt{\epsilon})^{2}}}{C}$ because it leads to a negative value for $\sigma$; see the expression for $\sigma$ below. In this case, the dual problem is infeasible and the primal is unbounded since it cannot be infeasible. The condition $C(\kappa+$ $\sqrt{\epsilon})^{2}>A C-B^{2}$ is equivalent to

$$
\epsilon>\left(\sqrt{A-B^{2} / C}-\sqrt{\theta /(1-\theta)}\right)^{2}
$$

[we do not allow $\epsilon=\left(\sqrt{A-B^{2} / C}-\sqrt{\theta /(1-\theta)}\right)^{2}$ since it results in a $\sigma$ that grows without bound, hence the problem is unbounded]. Using the above expression for $\lambda$ in the equation $e^{T} x=1$ we solve for $\sigma$ to get

$$
\sigma=\frac{\kappa+\sqrt{\epsilon}}{\sqrt{B^{2}-A C+C(\kappa+\sqrt{\epsilon})^{2}}} .
$$

Substituting $\lambda$ and $\sigma$ to the expression for $x$ obtained from the first-order conditions we obtain the solution 


$$
x^{*}=\frac{1}{\sqrt{C(\kappa+\sqrt{\epsilon})^{2}-\Delta}} \Gamma^{-1} \mu^{\mathrm{nom}}+\left[-\frac{B}{C \sqrt{C(\kappa+\sqrt{\epsilon})^{2}-\Delta}}+\frac{1}{C}\right] \Gamma^{-1} e,
$$

where we have defined $\Delta=A C-B^{2}$ for simplicity. The portfolio problem for robust $\mathrm{VaR}$ under distribution and ellipsoidal mean return ambiguity is resolved similarly. In fact, the only change is in the definition of $\kappa$. Therefore, we have proved the following result.

\section{Theorem 5}

1. The distribution and mean return ambiguity robust CVaR portfolio choice, i.e., the solution to problem

$$
\min _{x \in \mathbb{R}^{n}: e^{T} x=1} \max _{\bar{\mu} \in U_{\bar{\mu}}} \max _{\pi \in \mathbb{D}} \operatorname{CVaR}_{\theta}(x)
$$

is given by

$$
x^{*}=\frac{1}{\sqrt{C(\kappa+\sqrt{\epsilon})^{2}-\Delta}} \Gamma^{-1} \mu^{\mathrm{nom}}+\left[-\frac{B}{C \sqrt{C(\kappa+\sqrt{\epsilon})^{2}-\Delta}}+\frac{1}{C}\right] \Gamma^{-1} e,
$$

where $\Delta=A C-B^{2}, A=\left(\mu^{\mathrm{nom}}\right)^{\mathrm{T}} \Gamma^{-1} \mu^{\mathrm{nom}}, C=e^{\mathrm{T}} \Gamma^{-1} e, B=e^{\mathrm{T}} \Gamma^{-1} \mu^{\mathrm{nom}}, \quad$ and $\kappa=\sqrt{\theta /(1-\theta)}$ provided that $\epsilon>\left(\sqrt{A-B^{2} / C}-\kappa\right)^{2}$. If $\epsilon \leq\left(\sqrt{A-B^{2} / C}-\kappa\right)^{2}$ the problem is unbounded.

2. The distribution and mean return ambiguity robust VaR portfolio choice, i.e., the solution to problem

$$
\min _{x \in \mathbb{R}^{n}: e^{\mathrm{T} x} x=1} \max _{\bar{\mu} \in U_{\bar{\mu}}} \frac{2 \theta-1}{2 \sqrt{\theta(1-\theta)}} \sqrt{x^{\mathrm{T}} \Gamma x}-x^{\mathrm{T}} \bar{\mu}
$$

is given by

$$
x^{*}=\frac{1}{\sqrt{C(\kappa+\sqrt{\epsilon})^{2}-\Delta}} \Gamma^{-1} \mu^{\mathrm{nom}}+\left[-\frac{B}{C \sqrt{C(\kappa+\sqrt{\epsilon})^{2}-\Delta}}+\frac{1}{C}\right] \Gamma^{-1} e .
$$

where $\Delta=A C-B^{2}, A=\left(\mu^{\mathrm{nom}}\right)^{T} \Gamma^{-1} \mu^{\mathrm{nom}}, C=e^{\mathrm{T}} \Gamma^{-1} e, B=e^{\mathrm{T}} \Gamma^{-1} \mu^{\mathrm{nom}}, \quad$ and $\kappa=\frac{2 \theta-1}{2 \sqrt{\theta(1-\theta)}}$ provided that $\epsilon>\left(\sqrt{A-B^{2} / C}-\kappa\right)^{2}$. If $\epsilon \leq\left(\sqrt{A-B^{2} / C}-\kappa\right)^{2}$ the problem is unbounded.

Notice that the optimal portfolios are mean-variance efficient. Using the data in Chen et al. (2011) we plot the optimal CVaR function $\sqrt{\frac{\theta}{1-\theta}} \sqrt{x^{\mathrm{T}} \Gamma x}-x^{\mathrm{T}} \mu^{\mathrm{nom}}+$ $\sqrt{\epsilon} \sqrt{x^{\mathrm{T}} \Gamma x}$ evaluated at $x^{*}$ as a function of $\epsilon$ in Fig. 3 for two different values of $\theta$. As the confidence parameter $\epsilon$ increases (i.e., confidence in the mean return estimate diminishes), the optimal robust $\mathrm{CVaR}$ increases, which implies an increase in risk (Fig. 3). A similar behavior occurs for robust $\mathrm{VaR}$ optimal value function. 
Interestingly the optimal robust $\mathrm{CVaR}$ and $\mathrm{VaR}$ increase for constant $\epsilon$ when the quantile parameter $\theta$ increases (Fig. 3). When the uncertainty set $U_{\bar{\mu}}$ is reduced to a single point $\mu^{\text {nom }}=\mu$, i.e., for $\epsilon=0$ we obtain precisely the result of Chen et al. (2011), namely, Theorem 2.9:

\section{Corollary 1}

1. The distribution ambiguity robust CVaR portfolio choice, i.e., the solution to problem

$$
\min _{x \in \mathbb{R}^{n}: e^{T} x=1} \max _{\pi \in \mathbb{D}} \operatorname{CVaR}_{\theta}(x)
$$

is given by

$$
x^{*}=\frac{1}{\sqrt{C \kappa^{2}-\Delta}} \Gamma^{-1} \mu+\left[-\frac{B}{C \sqrt{C \kappa^{2}-\Delta}}+\frac{1}{C}\right] \Gamma^{-1} e .
$$

where $\Delta=A C-B^{2}, A=\mu^{\mathrm{T}} \Gamma^{-1} \mu, C=e^{\mathrm{T}} \Gamma^{-1} e, B=e^{\mathrm{T}} \Gamma^{-1} \mu$, and $\kappa=\sqrt{\theta /(1-\theta)}$ provided that $\theta /(1-\theta)>A-B^{2} / C$. If $\theta /(1-\theta) \leq A-B^{2} / C$ the problem is unbounded.

2. The distribution ambiguity robust VaR portfolio choice, i.e., the solution to problem

$$
\min _{x \in \mathbb{R}^{n}: e^{\mathrm{T}} x=1} \frac{2 \theta-1}{2 \sqrt{\theta(1-\theta)}} \sqrt{x^{\mathrm{T}} \Gamma x}-x^{\mathrm{T}} \mu
$$

is given by

$$
x^{*}=\frac{1}{\sqrt{C \kappa^{2}-\Delta}} \Gamma^{-1} \mu+\left[-\frac{B}{C \sqrt{C \kappa^{2}-\Delta}}+\frac{1}{C}\right] \Gamma^{-1} e .
$$

where $\Delta=A C-B^{2}, A=\mu^{\mathrm{T}} \Gamma^{-1} \mu, C=e^{\mathrm{T}} \Gamma^{-1} e, B=e^{\mathrm{T}} \Gamma^{-1} \mu$, and $\quad \kappa=\frac{2 \theta-1}{2 \sqrt{\theta(1-\theta)}}$ provided that $\left(\frac{2 \theta-1}{2 \sqrt{\theta(1-\theta)}}\right)^{2}>A-B^{2} / C$. If $\left(\frac{2 \theta-1}{2 \sqrt{\theta(1-\theta)}}\right)^{2} \leq A-B^{2} / C$ the problem is unbounded.

Acknowledgments The revised version of the paper benefited greatly from the comments of an anonymous referee.

\section{References}

Ben-Tal A, Nemirovski A (1999) Robust solutions to uncertain linear programming problems. Oper Res Lett 25(1):1-13

Ben-Tal A, Nemirovski A (1998) Robust convex optimization. Math Oper Res 23(4):769-805

Ben-Tal A, Teboulle M (2007) An old-new concept of convex risk measures: the optimized certainty equivalent. Math Finance 17:449-476

Best MJ, Grauer R (1991) Sensitivity analysis for mean-variance portfolio problems. Manag Sci 37(8):980-989

Best MJ, Grauer RR (1991) On the sensitivity of mean-variance-efficient portfolios to changes in asset means: some analytical and computational results. Rev Financial Stud 4(2):315-342 
Best MJ (2010) Portfolio optimization, financial mathematics series. Chapman \& Hall/CRC, Boca Raton

Black F, Litterman R (1992) Global portfolio optimization. Financial Anal J 48:28-43

Chen L, He S, Zhang S (2011) Tight bounds for some risk measures, with applications to robust portfolio selection. Oper Res 59(4):847-865

Delage E, Ye Y (2010) Distributionally robust optimization under moment uncertainty with application to data-driven problems. Oper Res 58(3):596-612

El Ghaoui L, Oks M, Oustry F (2003) Worst-case value-at-risk and robust portfolio optimization: a conic programming approach. Oper Res 51(4):543-556

Garlappi L, Uppal R, Wang T (2007) Portfolio selection with parameter and model uncertainty: a multiprior approach. Rev Financial Stud 20(1):41-81

Goldfarb D, Iyengar G (2003) Robust portfolio selection problems. Math Oper Res 28(1):1-38

Ling A, Xu C (2012) Robust portfolio selection involving options under a "marginal + joint" ellipsoidal uncertainty set. J Comput Appl Math 236:3373-3393

Natarajan KD, Sim M, Uichanco J (2010) Tractable robust expected utility and risk models for portfolio optimization. Math Finance 20(4):695-731

Popescu I (2005) Robust mean-covariance solutions for stochastic optimization. Oper Res 55(1):98-112

Rockafellar RT, Uryasev S (2000) Optimization of conditional value-at-risk. J Risk 2(3):21-41

Rockafellar RT, Uryasev S (2002) Conditional value-at-risk for general loss distributions. J Bank Finance 26:1443-1471

Shapiro A (2011) Topics in stochastic programming, CORE lecture series. Université catholique de Louvain, Louvain-la-neuve

Steinbach M (2001) Markowitz revisited: mean-variance models in financial portfolio analysis. SIAM Rev 43(1): 31-85

Tong X, Qi L, Wu F, Zhou H (2010) A smoothing method for solving portfolio optimization with CVaR and applications in allocation of generation asset. Appl Math Comput 216:1723-1740

Zhu S, Fukushima M (2009) Worst-case conditional value-at-risk with application to robust portfolio management. Oper Res 57(5):1155-1168 